vor diesem Hintergrund mit drei knappen Seiten deutlich zu kurz.

Weiterhin fällt auf, dass Forster ihre begrifflichen Grundlagen nur unzureichend klärt. Dem in wissenschaftlichen Debatten umstrittenen Problems der Definition von Terrorismus widmet Forster beispielsweise sechs Seiten (25ff) - verweist hierbei aber nur auf die in britischen Gesetzen zu findende Definition, ohne diese einzuordnen. Auch eine Klärung oder Diskussion des Begriffes Freiheit findet sich lediglich ansatzweise in zitierten Urteilspassagen, eine eigenständige Auseinandersetzung hierzu vermisst der kritische Leser. Neben diesen fehlenden Begriffsdefinitionen werden auch juristische Fachbegriffe nicht immer einheitlich verwendet. So wird beispielsweise die Festnahme mit anschließendem Polizeigewahrsam zuweilen Detention without charge, an anderer Stelle aber auch Detention without trial genannt (250ff), ohne näher zu erläutern, wofür diese sprachliche Unterscheidung nötig ist. Schlussendlich wäre aus politikwissenschaftlicher Sicht vorteilhaft gewesen, wenn sich die Autorin an den passenden Stellen etwas ausführlicher mit den zugrunde liegenden rechts- und vor allem politikwissenschaftlichen Theorien beschäftigt hätte.

Diese Schwächen sind jedoch nur kleine Einschränkungen einer ansonsten soliden rechtswissenschaftlichen Arbeit. Für die Politikwissenschaft stellt die vorliegende Untersuchung vor allem bei weitergehenden Arbeiten zur Anti-Terror-Politik des Vereinigten Königreiches, aber auch zum britischen Rechtssystem ein vielfältiges Reservoir mit umfangreichen Informationen und Verweisen dar.
Hensell, Stephan. Die Willkür des Staates. Herrschaft und Verwaltung in Osteuropa. Wiesbaden. VS Verlag 2009. 256 Seiten. 39,90€.

Wer des Mehltaus der defekten Demokratisierungsliteratur überdrüssig ist, wird Hensells Buch zunächst wie einen frischen Espresso begrüßen. Statt zu verkünden, wie theoriewidrig sich „defekte" postsozialistische Staaten verhalten, ist Hensell an den tatsächlichen Herrschaftspraktiken interessiert. Die im Jahre 2008 an der HU Berlin abgeschlossene Dissertation richtet ihr Augenmerk auf die Erblasten des sozialistischen Staates und die Operationsweise des spät- bzw. postsozialistischen Regierens. Hensell begründet sein Thema mit der defizitären Analyse des bürokratischen Staates, den merklichen Disproportionen bei der Auswahl osteuropäischer Untersuchungsfälle und der „mangelnden Historizität" von Transformationsforschung. Er ordnet seine Arbeit in die alt-neuen Diskussionen zu „moderner Staatlichkeit" ein und identifiziert sich als Vertreter der „Pfadabhängigkeit“.

Über „den“ postsozialistischen Staat wirft Hensell das von Max Weber und Pierre Bourdieu geknüpfte Begriffsnetz. Etwa die Hälfte des Buches besteht so aus einer Rekapitulation von Webers Aussagen zum patrimonialen Staat und zur personalisierten Herrschaft sowie aus Bourdieus Trifolium aus „Feld“, „Kapital“ und „Habitus“. Der sozialistische Patrimonialismus zeichnete sich laut Hensell durch mangelnde Trennung öffentlicher und privater Sphären, Personalisierung und Klientelismus aus. Obschon eine Form der Modernisierung, gelang es dem Sozialismus Hensell zufolge nicht, die traditionelle Beziehungen zu überwinden - mit hybriden Herrschaftsformen als Folge. 
Der empirische Teil der Arbeit - etwa ein Drittel der Studie - untersucht die Polizeipraktiken in Albanien und Georgien. Hensells Fallstudien entstanden in den Jahren 2003-2006 auf der Basis von zahlreichen Interviews mit albanischen und georgischen Polizisten. Für Albanien konstatiert er die Dominanz von zwei sich abwechselnden Patronageparteien, die während der jeweiligen Amtszeit die öffentliche Verwaltung, darunter die Polizei, kapern. Die albanischen Gesetzeshüter seien in den lokalen Schmuggel verwickelt und mit „kriminellen Sphären“ verzahnt (162). In Georgien wiederum beobachtet er eine schwache Steuerbasis, die zu Praktiken der Selbstfinanzierung im öffentlichen Dienst führt, darunter Ämterkäuflichkeit bei der Polizei, sowie zur Verflechtung des Innenministeriums mit diversen Firmen (199). Aufgrund der klammen öffentlichen Haushalte, so Hensell, wird der georgische Gendarm zum Räuber (182f.). Der patrimoniale Staat, so möchte man schließen, ist vor allem Ausdruck eines Mangels - und nicht des „Fluchs der Ressourcen“.

Unter den Typen des Spätsozialismus beschränkt sich Hensell auf einen, nämlich den patrimonialen Typus. Die pauschale Aussage, dass „die Staaten in Osteuropa [...] sich daher als bürokratisch-patrimonial charakterisieren “ lassen (207) verallgemeinert ungebührlich, dafür ist die Fallauswahl zu gering. Zudem werden nur zwei vergleichsweise ähnliche Fälle untersucht. Die Makrobegiffe von Weber und Bourdieu werden als Setzungen, bisweilen gar wie Satzungen vorgetragenen und für den Fallvergleich nicht hinreichend operationalisiert. Von daher bleibt das Potenzial des Fallvergleichs suboptimal. Die Weberschen und Bourdieuschen Begriffe wären eher geeignet gewesen, neue Erkenntnisse hervorzubringen, wenn sie selbst als ergebnisoffene, d. h. falsifizierbare, Hypothesen formuliert worden wären. Zudem gibt der Autor durchaus zu, dass „die hier verwendeten Begrifflichkeiten nur eine grobe Benennung und Einordnung der faktisch eine große Vielzahl aufweisenden Praktiken ermöglichte" (213). Hensell ist sich in seinen Schlussfolgerungen der begrenzten Reichweite seiner empirischen Aussagen und staatstheoretischen Schlussfolgerungen durchaus bewusst.

Hensells anregende, konzise und theoretisch ambitionierte Dissertation schließt an die zahlreichen Studien zum Staatscharakter und den neo-patrimonialen Herrschaftspraktiken im subsaharischen Afrika an. Während Hensell die normative Transformationsforschung schneidend kritisiert, besteht eine ähnliche Gefahr bei der Vermessung des postsozialistischen Staates anhand eines idealtypischen (Weberschen) Modells des rationalen „Anstaltstaates“. Die Stärke der Arbeit liegt zweifellos in der Reflexion über die wechselseitige Beeinflussung von patrimonialen und rationalen Herrschaftspraktiken, in den detaillierten Einblicken in die Polizeimilieus in Georgien und Albanien und der Formulierung einer künftigen Forschungsagenda.

Andreas Heinemann-Grüder

\section{Huhnholz, Sebastian. Dschihadistische Raumpraxis. Raumordnungspolitische Herausforderungen des militanten sunnitischen Fundamentalismus.} Berlin. LIT Verlag 2010. 168 Seiten. $24,90 €$.

Dieses mit einem Humboldt-Preis ausgezeichnete Buch nimmt unter den im- 